\title{
Forecasting Gas and Oil Potential of Subsoil Plots via Co- analysis of Satellite, Geological, Geophysical and Geochemical Information by Means of Subjective Logic
}

\author{
MYKHAILO O. POPOV, MAKSYM V. TOPOLNYTSKYI, OLGA V. TITARENKO, \\ SERGEY A. STANKEVICH, ARTEM A. ANDREIEV \\ Scientific Centre for Aerospace Research of the Earth \\ National Academy of Sciences of Ukraine \\ 55-B Oles Gonchar str., Kiev, 01054, \\ UKRAINE
}

\begin{abstract}
The purpose of the paper is to substantiate a new approach to forecasting hydrocarbon potential of subsoil plots via co-analysis of satellite, geo-geophysical and geochemical information by means of subjective logic. Basic concepts of subjective logic are given, the method of forecasting the oil and gas potential of a subsoil plots is described. The method is tested by applying it to real area with hydrocarbon deposits.

Key-Words: Hydrocarbon deposit, Satellite, Heterogeneous information, Co-analysis, Conflicting hypotheses, Uncertainty, Subjective logic
\end{abstract}

Received: April 5, 2020. Revised: August 1, 2020. Accepted: August 6, 2020. Published: August 18, 2020

\section{Introduction}

For over a century, hydrocarbons have remained one of the most common sources of energy, and every year humanity's demand for oil and natural gas is constantly growing. Despite increased use of renewable energy sources and increased energy efficiency, demand for oil and gas continues to grow along with energy consumption in most regions of the world. For example, the annual report of the International Association of Oil and Gas Producers [1] states that the demand for oil in 2018 increased by $30 \%$ compared to 2000 , and the demand for natural gas increased even more - by $60 \%$ compared to the same period.

It should be said that the 2020 economic crisis caused by the SARS-CoV-2 coronavirus has greatly changed the situation in the world oil and gas market, but it is obviously that this is a transient phenomenon. Understanding this fact and looking ahead, oil and gas producing countries do not reduce, in general, the volumes of work on exploring new hydrocarbon deposits.

However, the world experience shows, the effectiveness of search of new hydrocarbon deposits is still not high enough, and the positive conclusions according the availability of hydrocarbons which are provided by experts on the basis of studying the subsoil plots properties are unfortunately often not confirmed by the results of drilling [2].
The main reasons for the erroneous conclusions are the insufficient knowledge of the genesis of hydrocarbon formation, the ambiguity of the existing forecast criteria for hydrocarbon accumulations and their dependence on geological conditions and occurrence depths, the low efficiency of existing direct methods for hydrocarbon deposits prospecting, the presence of artifacts, etc. [3].

Satellite technologies occupy an important place in hydrocarbon prospecting, since they give a possibility promptly to receive important information about landcover and terrain in whole $[4,5]$. Thus, a procedure for predicting the oil and gas potential of subsoil plots requires the utilization of a large amount of heterogeneous data that have different accuracy and completeness, differ in presentation forms, spatial scale, the moment of assessment or observation, etc.

Analysis of heterogeneous and incomplete data is a rather complicated process; therefore, to solve the problem, artificial intelligence methods, algorithmic decision-making procedures, etc. are used [6].

The purpose of the paper is to develop a new approach to forecasting the oil and gas potential of subsoil plots. This approach actively exploits the mathematical apparatus of subjective logic developed by A. Jøsang [7], and its implementation allows you to: 
- adjust the subjectivity and uncertainty of expert judgments,

- generate adequate on the results of the analysis one-time or rare events;

- avoid the majority of logical-computational problems that usually arise when combining conflicting hypothesis and opinions.

In total, these advantages provide a good opportunity to increase the reliability of forecasting gas and oil potential of subsoil plots.

The paper is structured as follows. Section 2 examines state of the problem. Section 3 describes basic concepts of the subjective logic [7]. In section 4, the new method to forecast gas and oil potential of subsoil plots through co-analysis of satellite, geological, geophysical and geochemical information by means of subjective logic is proposed and described in detail. In section 5, we conduct validation of the proposed method on real data. And, finally, in section 6, conclusions and directions for further research are represented.

\section{State of the problem}

The intellectualization of data processing is based on the creation and the implementation of mathematical (formal) models of objects and causal relationships between them. The formal models allow to algorithmize the hydrocarbons prospecting process and, as a consequence, to reduce, at least in part, the level of subjectivity of forecast estimates and make them more adequate and reliable. A confirmation of this fact is the successful application of expert systems PROSPECTOR, Dipmeter Advisor, GEOPLAY, but not only [8].

It is known that most of the known models and algorithms for data processing and decisionmaking are constructed using a probabilisticstatistical approach. However, in the tasks of remote forecasting of the oil and gas potential of subsoil plots, relying on a probabilistic paradigm is not the good solution.

Firstly, in most cases of studying subsurface areas, the data obtained are at least partially inaccurate and ambiguous, and the amount of available information is limited. In such cases, it is difficult to talk about statistically reliable solutions.

Secondly, despite significant progress in geological science, many issues of oil and gas genesis and of the influence of the deposit presence on soil and vegetation characteristics remain open and debatable.

Due to these reasons, expert judgments about the oil and gas potential of the subsoil usually carry a significant element of uncertainty and are often even contradictory. However, is known the processing of conflicting data by methods of probability theory is complex and can lead to incorrect results [9].

Therefore, new approaches to search, forecasting oil and gas potential of subsoil plots, based on other theoretical models are being developed. In the monography [10], a methodology for forecasting hydrocarbon potential of subsoil plots, based on the algorithmization of the processes of analysis of satellite, geological, geophysical and geochemical information is described. In [11], the method using the apparatus of fuzzy sets and fuzzy logic for of oil and gas prospecting sections of the Dnieper-Donetsk depression is developed. In [12], the technology of use of remotely-sensed data for quantitative estimate the potential of petroleum, natural gas and minerals taking into account the specific features of area is developed. In [13], it is proposed to detect intersection nodes of fault lines that are promising for hydrocarbon accumulation using the mathematical tools of the Dempster-Schafer evidence theory [14].

Authors propose a new approach for forecasting gas and oil potential of subsoil plots via co-analysis of satellite, geological, geophysical and geochemical information. The proposed approach relies on subjective logic, mathematic apparatus of which allows operating with uncertain and incomplete data, simple hypotheses and their disjunctions, as well as to combine conflicting hypotheses and opinions.

\section{Basic concepts}

Suppose $\Theta$ is a set of mutually exclusive and collectively exhaustive hypotheses:

$$
\Theta=\left\{H_{1}, H_{2}, \ldots, H_{j}, \ldots H_{J}\right\}
$$

and denote as $2^{|\theta|}$ a set, containing all subsets of different hypotheses:

$$
\begin{aligned}
& 2^{|\Theta|}=\left\{\varnothing,\left\{H_{1}\right\},\left\{H_{2}\right\}, \ldots,\left\{H_{j}\right\}, \ldots\left\{H_{J}\right\},\right. \\
& \left.\quad\left\{H_{1} \cup H_{2}\right\}, \ldots,\left\{H_{1} \cup H_{2} \cup \ldots \cup H_{J}\right\}, \Theta\right\},
\end{aligned}
$$

where $\varnothing$ denotes an empty set.

The set $\Theta$ is called Frame of Discernment (FoD); elements $H_{j} \mid j=1,2, \ldots, J$ of this set are called simple hypotheses. The set $2^{|\Theta|}$ is called Power Set; its subsets in the form of a disjunction of two or more hypotheses are called compound hypotheses.

Each subset $x \mid x \in 2^{|\Theta|}$ is associated with a corresponding number $m_{\Theta}(x)$, that satisfies conditions: 


$$
\left\{\begin{array}{l}
m_{\Theta}(x) \geq 0 \\
m_{\Theta}(\varnothing)=0 \\
\sum_{x \in 2^{|\Theta|}} m_{\Theta}(x)=1
\end{array}\right.
$$

The number $m_{\Theta}(x)$ is called belief mass for the subset $x$. If $m_{\Theta}(x)>0$ the subset $x$ is called focal one. The set of belief masses for all elements of the set $2^{|\Theta|}$ forms the Belief Mass Assignment (BMA), which is indicated as $m_{\Theta}(\Theta)$.

In subjective logic, for given $x \mid x \in 2^{|\Theta|}$ the following functions are introduced:

belief function

$$
b(x)=\sum_{y \subseteq x} m_{\Theta}(y) ; x, y \in 2^{|\Theta|}
$$

disbelief function

$$
d(x)=\sum_{y \cap x=\varnothing} m_{\Theta}(y) ; x, y \in 2^{|\Theta|}
$$

uncertainty function

$$
u(x)=\sum_{\substack{y \cap x \neq \varnothing \\ y \notin x}} m_{\Theta}(y) ; x, y \in 2^{|\Theta|}
$$

The sum of the belief, disbelief, and uncertainty values is equal 1 :

$$
b(x)+d(x)+u(x)=1 ; x \in 2^{|\Theta|} ; x \neq \varnothing
$$

As noted above, the set $2^{|\theta|}$ can include both simple and compound elements-hypotheses. Let $x$ be particular subset of set $2^{|\Theta|}$, then the number of elements it contains, is called atomicity of $x$ and noted by $|x|$. With help of the atomicity concept, one can estimate the degree of overlap that exists between any two subsets of the set $2^{|\Theta|}$.

Suppose $x, y \in 2^{|\Theta|}$, then for any given $y \neq \varnothing$ the relative atomicity of $x$ to $y$ is defined as

$$
a\left(\frac{x}{y}\right)=\frac{|x \cap y|}{|y|} ; x, y \in 2^{|\Theta|} ; y \neq \varnothing
$$

Note that $x \cap y=\varnothing \Rightarrow a\left(\frac{x}{y}\right)=0$ and $x \subseteq y \Rightarrow a\left(\frac{x}{y}\right)=1$. That's, relative atomicity lies within the $[0,1]$ interval. The relative atomicity of the atomic element (state) to its FoD, denoted by $a\left(\frac{x}{\Theta}\right)$, is written as $a(x)$. Unless otherwise stated, the relative atomicity of an element (state) is referred to the FoD.

The subjective logic also defines for given element $x$ the probability expectation function

$$
E(x)=\sum_{y} m_{\Theta}(y) \cdot a\left(\frac{x}{y}\right) ; x, y \in 2^{|\Theta|}
$$

Quite often, a researcher is not interested in all elements of the FoD $\Theta$, but only in one particular element. In such cases, in order to simplify the calculations, it is proposed in subjective logic to derive from an initial FoD $\Theta$ a binary $\operatorname{FoD} \tilde{\Theta}^{x}$ capable the following properties [15]:

A FoD $\tilde{\Theta}^{x}$ contains only two atomic elements: element of interest $x$ and $\neg x$ where $\neg x$ is the complement of $x$ in the FoD $\Theta$.

The BMA $m_{\tilde{\Theta}^{x}}$ on binary FoD $2^{|\tilde{\Theta}|}$ is defined according to:

$$
\begin{aligned}
& m_{\tilde{\Theta}^{x}}(x)=b(x) \\
& m_{\tilde{\Theta}^{x}}(\neg x)=d(x) \\
& m_{\tilde{\Theta}^{x}}\left(\tilde{\Theta}^{x}\right)=u(x)
\end{aligned}
$$

A binary FoD $\tilde{\Theta}^{x}$ with the BMA $m_{\tilde{\Theta}^{x}}$ that satisfies (10) is called the focused FoD with focus on $x$. The focused relative atomicity of element $x$ is defined by the following equation:

$$
\left\{\begin{array}{l}
a_{\tilde{\Theta}^{x}}(x)=\frac{E(x)-b(x)}{u(x)}, \quad \text { if } u(x) \neq 0 \\
a_{\tilde{\Theta}^{x}}(x)=a(x), \quad \text { if } u(x)=0
\end{array}\right.
$$

It should be emphasized that the belief, disbelief and uncertainty functions of $x$ are identical in set $2^{|\Theta|}$ and set $2^{|\tilde{\theta}|}$. The focused relative atomicity is also defined so that the probability expectation value for the element $x$ is the same in the sets $\Theta$ and $\tilde{\Theta}^{x}$.

The operation of transforming a FoD $\Theta$ in a binary set $\tilde{\Theta}^{x}$ is useful in situations when it is necessary to combine hypotheses.

\section{The method}

Our ultimate purpose is to develop the method for assessing the oil and gas potential of every plot within the given area. Structural and logical scheme of the proposed method is shown in Fig. 1. 


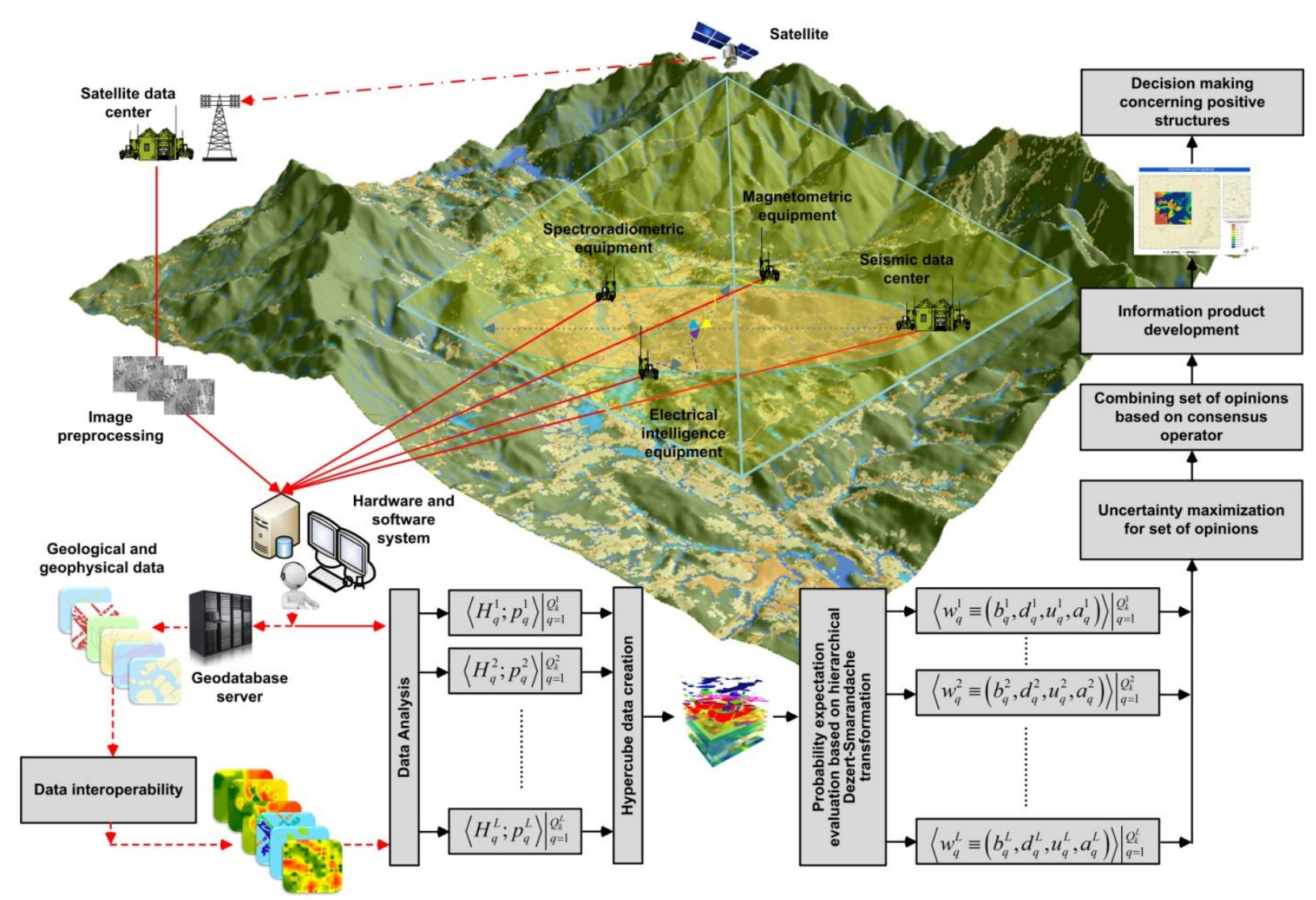

Fig. 1. Structural and logical scheme of the proposed method

The method is a multi-step one, the work begins with the collection of data on the study area and should end with the creating an information product in the form of a map with the division of the plots on classes in accordance with their forecasted oil and gas potential.

Step 1. Data collection and preparation. The amount of data required is large enough, so usually all available technical and information sources are exploit, including:

- equipment for seismic survey;

- magnetometric equipment;

- electrical intelligence equipment;

- aerial and satellite imagery;

- geological survey data;

- spectroradiometric devices;

- humidity maps and data of soil geochemical analysis;

- topographic maps and maps of lineaments;

- digital elevation models, digital terrain models and many other.

Using technical means, the necessary observations and measurements are carried out, and they are planned in such a way as to obtain sufficient information on the spatial distribution of the required physical parameters (brightness, humidity, absorption, etc.).

Some part of the required information is obtained through geological funds, by searching the global telecommunication networks, etc. The spatial distribution of physical parameter over study area is considered as a separate information layer and together all layers create a hypercube.

It should be noted that the data obtained from different sources have different presentation structure, formats, differ in size, frequency of measurements, etc. [16]. Therefore, measures to ensure the interoperability of the collected data must be taken.

Step 2. Data Analysis. Roughly speaking, all plots of study area can be divided on two categories: having hydrocarbon potential (so named positive) ones and ones don't containing hydrocarbons (negative). As the positive plots are considered oil, natural gas and condensate collectors.

Therefore, initially we have four hypotheses regarding possible state of a plot:

$$
\begin{aligned}
& H_{1}-\text { oil; } \\
& H_{2} \text { - natural gas; }
\end{aligned}
$$


$\mathrm{H}_{3}$ - condensate;

$\mathrm{H}_{4}$ - there are no signs of hydrocarbons.

These four hypotheses are simple ones and together create the FoD.

The purpose of data analysis is to obtain for each plot hypotheses distribution and corresponding belief masses. Such distributions must be form separately and independently for each information layer.

Suppose, there are $K$ plots in the study area and as a result of the measurements and observations on step 1, and $L$ information layers number have been constructed. Hypotheses for the $k$ plot $(k=1,2, \ldots, K)$ obtaining by analysis of the data of the $l$ layer $(l=1,2, \ldots, L)$, will be indicated $H^{l}(k)$.

The set of hypotheses regarding the state of $k$ plot $(k=1,2, \ldots, K)$, which is formed on the basis of the analysis of the data of the $l$ layer, can be written as a tuple

$$
\left\langle H_{q}^{l}(k) ; m_{q}^{l}(k)\right\rangle \mid \begin{aligned}
& Q_{k}^{l} \\
& q=1
\end{aligned}
$$

where $H_{q}^{l}(k)$ is the $q^{\text {th }}$ hypothesis regarding the state of the $k$ plot; $m_{q}^{l}(k)$ is the belief mass of the hypothesis $H_{q}^{l}(k) ; Q_{k}^{l}$ is a total number of hypotheses regarding the state of the $k$ plot, which are formed on the basis of the data of the $l$ information layer.

It must be pointed out that the task of forming tuples (12) requires considerable intellectual effort; therefore, it is assumed that an expert method is used to solve it. In the set of hypotheses (12), which is formed for a plot, both simple and compound hypotheses can be. The next step is to form the opinion about the state of every plot of study area.

Step 3. Conversion of hypotheses into opinions.

Each simple hypothesis from the FoD is converted into the corresponding opinion. For this, based on the known belief masses of this hypothesis, the values of the belief, disbelief and uncertainty that determine this corresponding opinion must be calculated using expressions (4)(6).

Calculating the mathematical expectation of the hypothesis, it should be borne in mind that the correct transition from the initial set $\Theta$ to the binary set $\tilde{\Theta}^{x}$ is hindered by the interdependence between the probability expectation function $\mathrm{E}(\mathrm{x})$ and the focused relative atomicity $a_{\tilde{\Theta}^{x}}(x)$. This problem may be solved with help the Hierarchical DSmP transformation procedure proposed in [17]. Its essence is as follows.
Suppose there is the FoD $\Theta=\left\{H_{1}, H_{2}, H_{3}\right\}$ and a known the set

$$
\begin{aligned}
2^{|\Theta|}= & \left\{\left\langle H_{1} ; m_{1}\right\rangle,\left\langle H_{2} ; m_{2}\right\rangle,\left\langle H_{3} ; m_{3}\right\rangle,\right. \\
& \left\langle H_{12} ; m_{12}\right\rangle,\left\langle H_{13} ; m_{13}\right\rangle, \\
& \left.\left\langle H_{23} ; m_{23}\right\rangle,\left\langle H_{123} ; m_{123}\right\rangle\right\}
\end{aligned}
$$

in which each simple or compound hypothesis $H$ has own corresponding belief mass $\mathrm{m}$. Then the procedure of hierarchical probabilistic transformation can be explained with help of Fig. 2

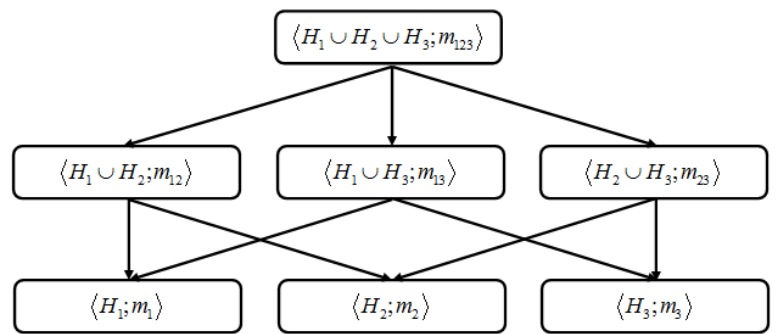

Fig. 2. Graphic illustration of Hierarchical DSmP transformation

Transformation process starts with the redistribution of the belief mass of the compound hypothesis $\left\langle H_{123} ; m_{123}\right\rangle \equiv\left\langle H_{1} \cup H_{2} \cup H_{3} ; m_{123}\right\rangle$ to the belief masses of the compound hypotheses $\left\langle H_{1} \cup H_{2} ; m_{12}\right\rangle, \quad\left\langle H_{1} \cup H_{3} ; m_{13}\right\rangle, \quad\left\langle H_{2} \cup H_{3} ; m_{23}\right\rangle$. This stage occurs by adding to their masses the product of mass $m_{123}$ of the compound hypothesis $H_{123}$ by the re-distribution coefficient, which characterizes the proportion of the corresponding mass in the total sum of masses $H_{12}, H_{13}$ and $H_{23}$.

In the second stage, the two-membered disjunctions are decomposed into singletons, and for each of them the belief mass value is determined in the same way. Re-distribution of masses between hypotheses in the given stages is carried out in accordance with the expression:

$$
\begin{aligned}
& H T_{D S m}(H(v-1))=m(H(v-1))+ \\
& +\sum_{\substack{H(v)>H(v-1) \\
H(v), H(v-1) \in 2^{|l|}}}[m(v)) \cdot R(H(v-1), H(v))
\end{aligned}
$$

where $R(H(v-1), H(v))$ is a proportional redistribution coefficient, which is defined as

$$
R(H(v-1), H(v))=\frac{m(H(v-1))}{\left.\sum_{\substack{H(v) \supset H(v-1) \\ H(v), H(v-1) \in 2^{||^{\mid}}}} m(H-1)\right)}
$$

where $v$ is a stage of transformation.

The described procedure is implemented into an algorithm 1 represented below. The algorithm provides the formation of an opinion space; in pseudocode, it is assumed that the total number of simple hypotheses is J. The hypotheses are formed for every plot of the study area in each layer 
separately and independently. Further, an opinion space is algorithmically formed from these hypotheses, i.e., the algorithm performs the transformation

$$
\begin{aligned}
& H_{j}^{l} \Leftrightarrow w_{j}^{l}=\left(b_{j}^{l}, d_{j}^{l}, u_{j}^{l}, a_{j}^{l}\right) ; \\
& l=1,2, \ldots, L ; j=1,2, \ldots, J .
\end{aligned}
$$

Algorithm 1: The algorithm to generate an opinion sets

Input: Belief Mass Assignment

// Form as

$$
\begin{aligned}
& m_{l}=\left(\left\{H_{1}^{l} ; m_{1}^{l}\right\},\left\{H_{2}^{l} ; m_{2}^{l}\right\}, \ldots,\left\{H_{Q^{l}}^{l} ; m_{Q^{l}}^{l}\right\}\right. \text {, } \\
& l=1,2, \ldots, L, q=1,2, \ldots, Q^{l} \\
& \text { for } q \text { in } Q^{l} \text { : } \\
& b\left(H_{j}^{l}\right)=\sum_{H_{q}^{l} \subseteq H_{j}^{l}} m_{\Theta}\left(H_{q}^{l}\right) \\
& d\left(H_{j}^{l}\right)=\sum_{H_{q}^{l} \cap H_{j}^{l}=\varnothing} m_{\Theta}\left(H_{q}^{l}\right) \\
& u\left(H_{j}^{l}\right)=\sum_{\substack{H_{q}^{l} \sim H_{j}^{l} \neq \varnothing \\
H_{q}^{l} \subset H_{j}^{l}}} m_{\Theta}\left(H_{q}^{l}\right) \\
& E\left(H_{j}^{l}(v-1)\right)=m\left(H_{j}^{l}(v-1)\right)+ \\
& +\sum_{H_{q}^{l}(v) \supset H_{q}^{l}(v-1)}\left[m\left(H_{q}^{l}(v)\right) \cdot R\left(H_{q}^{l}(v-1), H_{q}^{l}(v)\right)\right. \\
& R\left(H_{j}^{l}(v-1), H_{j}^{l}(v)\right)=\frac{m(H(v-1))}{\sum_{\substack{H(v) \supset H(v-1) \\
H(v), H(v-1) \in 2^{|l|}}} m(H(v-1))}
\end{aligned}
$$$$
\text { if } u\left(H_{j}^{l}\right)=0
$$

$$
a\left(H_{j}^{l}\right)=1 / J
$$

else

$$
a\left(H_{j}^{l}\right)=\frac{E\left(H_{j}^{l}\right)-b\left(H_{j}^{l}\right)}{u\left(H_{j}^{l}\right)}
$$

end

Output: $w^{l}=\left(w_{1}^{l}, w_{2}^{l}, \ldots, w_{j}^{l}, \ldots, w_{J}^{l}\right)$;

$$
l=1,2, \ldots, L ; j=1,2, \ldots, J
$$

It should be noted that the opinions $w$ are determined by the belief masses of hypotheses $H$, which are estimated at step 2. Both estimates of the belief masses and decisions are usually carried out under conditions of partial uncertainty. Such conditions of data analysis adversely affect, in particular, the accuracy of the belief $b$ and disbelief $d$ values.

It was shown in [15] that under such conditions, belief $b$ and disbelief $d$ functions acquire greater adequacy if the uncertainty fractions that are present in these functions are exported into the uncertainty function $u$. This can be realized by adjusting the uncertainty function $\mathrm{u}$ values using the uncertainty maximization procedure.

Step 4. Adjustment of belief and disbelief functions into opinions. The essence of the uncertainty maximization procedure is as follows [18]. Suppose that $w_{j}=\left(b_{j}, d_{j}, u_{j}, a_{j}\right)$ is a definite opinion about a binary unique event. Then at known $b_{j}$ and $d_{j}$ uncertainty maximization of an opinion $w_{j}$ is carried out by its transformation into an opinion $\hat{w}_{j}=\left(\hat{b}_{j}, \hat{d}_{j}, \hat{u}_{j}, \hat{a}_{j}\right)$ as follows:

$$
\text { for } E\left(w_{j}\right) \leq a_{j}: \quad \text { for } E\left(w_{j}\right)>a_{j} \text { : }
$$

$$
\left\{\begin{array} { l } 
{ \hat { b } _ { j } = 0 } \\
{ \hat { d } _ { j } = 1 - u _ { j } - \frac { b _ { j } } { a _ { j } } } \\
{ \hat { u } _ { j } = u _ { j } + \frac { b _ { j } } { a _ { j } } } \\
{ \hat { a } _ { j } = a _ { j } }
\end{array} \left\{\begin{array}{l}
\hat{b}_{j}=1-u_{j}-\frac{d_{j}}{1-a_{j}} \\
\hat{d}_{j}=0 \\
\hat{u}_{j}=u_{j}+\frac{d_{j}}{1-a_{j}} \\
\hat{a}_{j}=a_{j}
\end{array}\right.\right.
$$

Thus, for any $k$ plot $(k=1,2, \ldots, K)$, a set of $L$ tuples of opinions (by the number of information layers) is formed:

$$
\begin{aligned}
& \left.\left\langle w_{j}^{1}(k)\right\rangle\right|_{j=1} ^{J_{k}^{1}}=\left\langle\hat{w}_{1}^{1}(k), \ldots, \hat{w}_{j}^{1}(k), \ldots, \hat{w}_{J}^{1}(k)\right\rangle ; \\
& \left.\left\langle w_{j}^{l}(k)\right\rangle\right|_{j=1} ^{J_{k}^{l}}=\left\langle\hat{w}_{1}^{l}(k), \ldots, \hat{w}_{j}^{l}(k), \ldots, \hat{w}_{J}^{l}(k)\right\rangle ; \\
& \left.\left\langle w_{j}^{L}(k)\right\rangle\right|_{j=1} ^{J_{k}^{L}}=\left\langle\hat{w}_{1}^{L}(k), \ldots, \hat{w}_{j}^{L}(k), \ldots, \hat{w}_{J}^{L}(k)\right\rangle
\end{aligned}
$$

The next task is to combine these $L$ tuples into a single tuple whose components will reflect the properties of the $k$ plot $(k=1,2, \ldots, K)$.

The algorithm for the uncertainty maximization procedure is presented below.

Algorithm 2: The algorithm of uncertainty maximization process

Input: opinion sets

// Form as $w^{l}=\left(w_{1}^{l}, w_{2}^{l}, \ldots, w_{j}^{l}, \ldots, w_{J}^{l}\right)$;

for $j$ in $J$ :

$$
l=1,2, \ldots, L ; j=1,2, \ldots, J
$$

if $E\left(w_{j}^{l}\right)>a_{j}^{l}$

$$
\begin{aligned}
& \hat{b}_{j}^{l}=1-u_{j}^{l}-\frac{d_{j}^{l}}{1-a_{j}^{l}} \\
& \hat{d}_{j}^{l}=0
\end{aligned}
$$


else if $E\left(w_{j}^{l}\right) \leq a_{j}^{l}$

$$
\begin{aligned}
& \hat{u}_{j}^{l}=u_{j}^{l}+\frac{d_{j}^{l}}{1-a_{j}^{l}} \\
& \hat{a}_{j}^{l}=a_{j}^{l}
\end{aligned}
$$

$$
\begin{aligned}
& \hat{b}_{j}^{l}=0 \\
& \hat{d}_{j}^{l}=1-u_{j}^{l}-\frac{b_{j}^{l}}{a_{j}^{l}} \\
& \hat{u}_{j}^{l}=u_{j}^{l}+\frac{b_{j}^{l}}{a_{j}^{l}} \\
& \hat{a}_{j}^{l}=a_{j}^{l}
\end{aligned}
$$

end

Output: $\hat{w}^{l}=\left(\hat{w}_{1}^{l}, \hat{w}_{2}^{l}, \ldots, \hat{w}_{j}^{l}, \ldots, \hat{w}_{J}^{l}\right)$;

$$
l=1,2, \ldots, L ; j=1,2, \ldots, J
$$

Step 5. Combining opinions. Opinion tuples for each plot are combined independently. We assume that for any plot we have $L$ tuples, each of which contains $J$ opinions. Tuples are combined sequentially: first, the tuples of opinions formed from data of the first $(l=1)$ and second $(l=2)$ information layers are combined. Further, the new tuple, which is obtained as a result of combining, is combined with the tuple previously formed from the data of the third $(l=3)$ information layer, and so on.

Consider the mathematical aspect of combining. Suppose there are two tuples: $\left\langle\hat{w}_{1}^{l}(k), \ldots, \hat{w}_{j}^{l}(k), \ldots, \hat{w}_{J}^{l}(k)\right\rangle$, $\left\langle\hat{w}_{1}^{l+1}(k), \ldots, \hat{w}_{j}^{l+1}(k), \ldots, \hat{w}_{J}^{l+1}(k)\right\rangle$,

and into them the opinions of the same name must be combined in pairs $\hat{w}^{l}=\left(b^{l}, d^{l}, u^{l}, a^{l}\right)$ and $\hat{w}^{l+1}=\left(b^{l+1}, d^{l+1}, u^{l+1}, a^{l+1}\right)$.

In subjective logic, the operation of combining opinions is denoted by a symbol $\oplus$ and is written as follows:

$$
w_{j}^{l, l+1}(k)=w_{j}^{l}(k) \oplus w_{j}^{l+1}(k)
$$

The result of combining $w^{l, l+1}(k)=$ $\left(b_{j}^{l, l+1}, d_{j}^{l, l+1}, u_{j}^{l, l+1}, a_{j}^{l, l+1}\right) \quad$ is called a consensus opinion, and its belief, disbelief, uncertainty functions, and atomicity are calculated using the following formulas [18]:

$$
\left\{\begin{array}{l}
b_{j}^{l, l+1}=\frac{b_{j}^{l} u_{j}^{l+1}+b_{j}^{l+1} u_{j}^{l}}{u_{j}^{l}+u_{j}^{l+1}-u_{j}^{l} u_{j}^{l+1}} \\
d_{j}^{l, l+1}=\frac{d_{j}^{l} u_{j}^{l+1}+d_{j}^{l+1} u_{j}^{l}}{u_{j}^{l}+u_{j}^{l+1}-u_{j}^{l} u_{j}^{l+1}} \\
u_{j}^{l, l+1}=\frac{u_{j}^{l} u_{j}^{l+1}}{u_{j}^{l}+u_{j}^{l+1}-u_{j}^{l} u_{j}^{l+1}} \\
a_{j}^{l, l+1}=\frac{a_{j}^{l} u_{j}^{l+1}+a_{j}^{l+1} u_{j}^{l}-\left(a_{j}^{l}+a_{j}^{l+1}\right) u_{j}^{l}}{u_{j}^{l}+u_{j}^{l+1}-2 u_{j}^{l} u_{j}^{l+1}}
\end{array}\right.
$$

The pseudo-code in Algorithm 3 illustrates the fusion process intuitively.

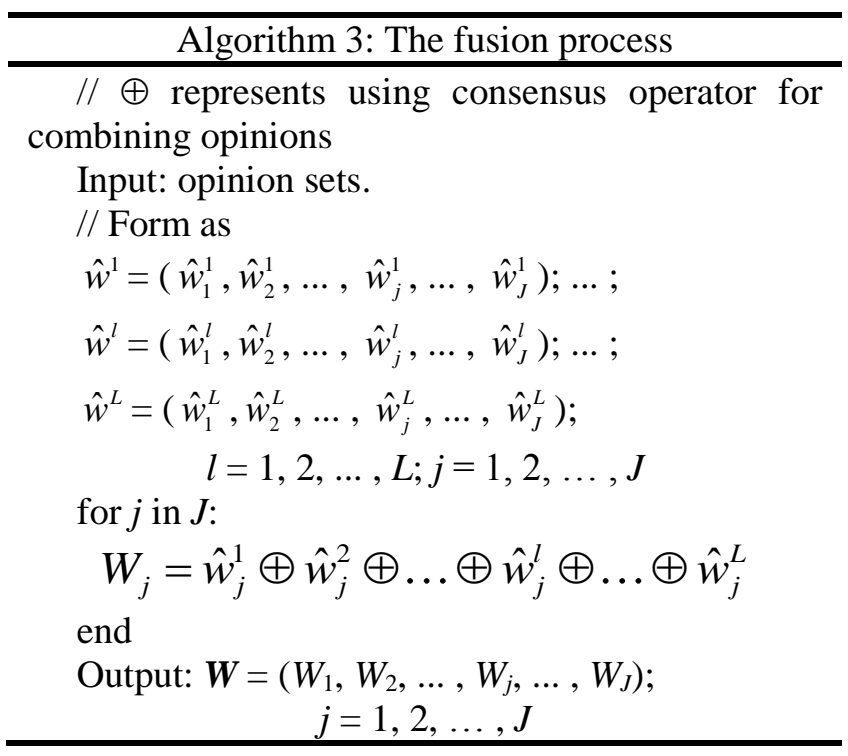

Further, the obtained consensus opinion $\left.\left\langle w_{j}^{l, l+1}(k)\right\rangle\right|_{j=1} ^{J_{k}^{l}}$ is combined with next opinion the tuple of (18). In total the operation of combining opinions is performed $L-1$ times, and as a result one tuple containing up to $J$ different opinions is calculated for the plot $\boldsymbol{W}=\left(W_{1}, W_{2}, \ldots, W_{j}, \ldots, W_{J}\right)$.

In the same way, the tuples for all plots are calculated.

As a rule, each tuple contains different consensus opinions about the oil and gas potential of the proper plot. It remains, to compare these opinions which each other and to choose one opinion that evaluates geological reality the most accurately.

Step 6. Forming final opinions on gas and oil potential of subsoil plots of the study area. The final assessment for each plot is obtained by a sequential pairwise analysis of all calculated opinions on its oil and gas prospects (see tuples of opinions in (18)). The procedure for comparing a 
pair of opinions and choosing the most preferred (most accurate) opinion is carried out in accordance with the following algorithm.

Let there be a pair of opinions, one of which is $W_{1}$, another is $W_{2}$. The $W_{1}$ opinion is characterized by $E\left(W_{1}\right), u\left(W_{1}\right), a\left(W_{1}\right)$ values, $W_{2}$ opinion is characterized by $E\left(W_{2}\right), u\left(W_{2}\right), a\left(W_{2}\right)$ values.

The procedure for a comparative analysis of these opinions begins with checking the conditions:

$$
\left|E\left(W_{1}\right)-E\left(W_{2}\right)\right| \leq \varepsilon_{E}
$$

where $\varepsilon_{E}=0.1 \min \left\{E\left(W_{1}\right), E\left(W_{2}\right)\right\}$.

If condition (21) is not satisfied, then choose is made in favor of the opinion characterized by a bigger value of $E(\cdot)$. If condition (21) is satisfied, then next condition is checked:

$$
\left|u\left(W_{1}\right)-u\left(W_{2}\right)\right| \leq \varepsilon_{u}
$$

where $\varepsilon_{u}=0.1 \min \left\{u\left(W_{1}\right), u\left(W_{2}\right)\right\}$.

If condition (22) is not satisfied, then the opinion with the biggest uncertainty value $u(\cdot)$ is choosed. Otherwise, the values $a\left(W_{1}\right)$ and $a\left(W_{2}\right)$ are compared and the opinion with the biggest relative atomicity value $a(\cdot)$ is choosed.

The described algorithm is sequentially applied to assess the oil and gas potential of each of the plots of the study area (Fig. 3).

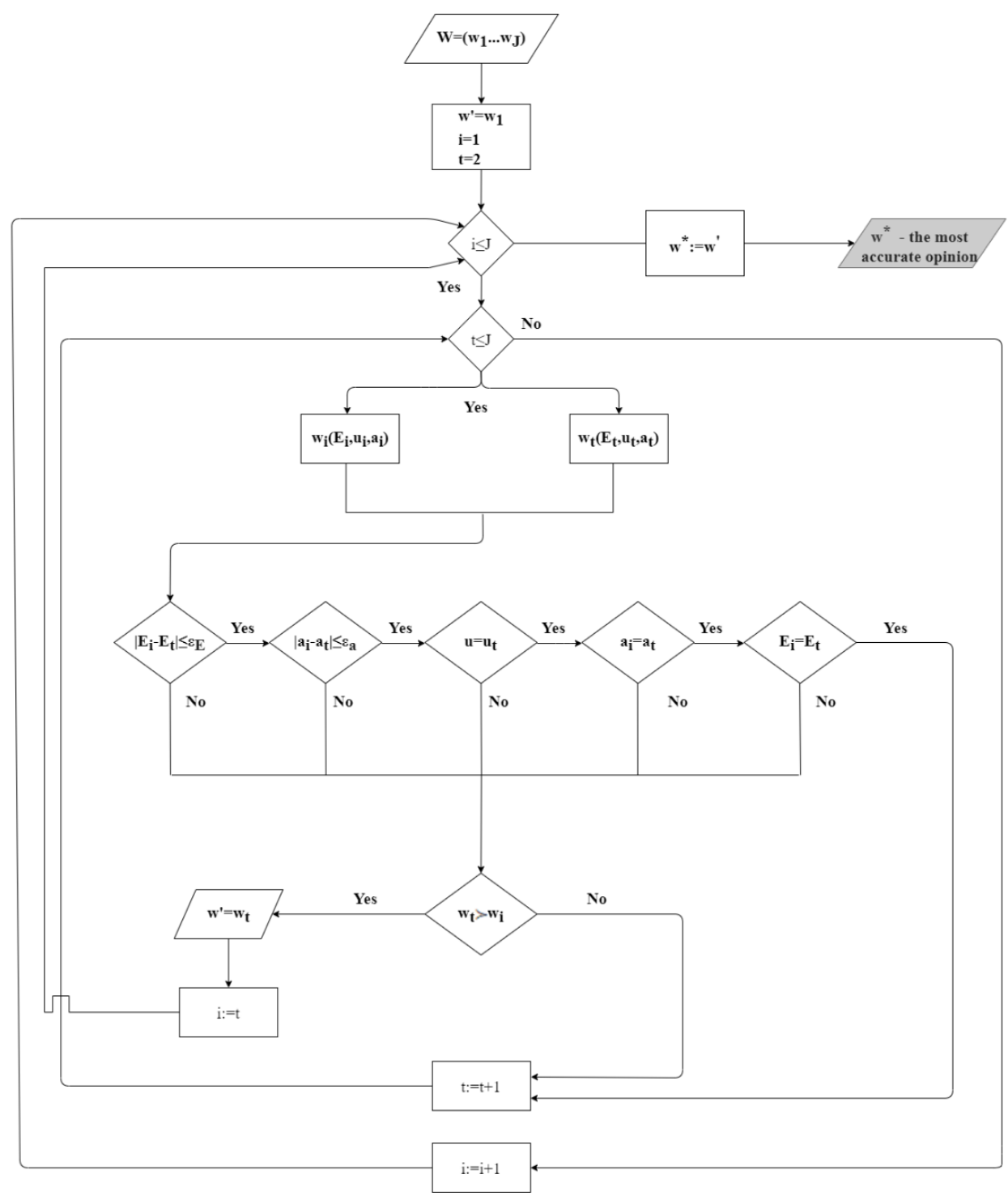

Fig. 3. Flowchart of the algorithm for the most accurate opinions determining regarding oil and gas potential of plots of the study area

Finally, according to the results of obtained unambiguous assessments of oil and gas potential of the plots, a map of oil and gas content for all study area is constructed. 
Loading the proposed method into a computer environment, one should take into account its quite big computational complexity. The greatest computational resources are required to implement Algorithm 3. The computational complexity of the matrix operations necessary for its implementation can be estimated using the expression $O(n, m)$ $2^{20} \times n \times m \times \log _{2} m$, where $m$ is the number of information layers, $n$ is information layer parameter. Calculations show that for $m=10$; $n=(255 \times 257) \sim 2^{16}$ (these figures are taken from the practical example below) we have $\mathrm{O}(\mathrm{n}, \mathrm{m}) \sim 2^{38}$ operations.

In general, the proposed method belongs to the class of tasks having the exponential computation complexity; however, recently in [22] a highly efficient exact dynamic programming algorithm was proposed allowing successfully to solve similar tasks.

\section{Testing the proposed method}

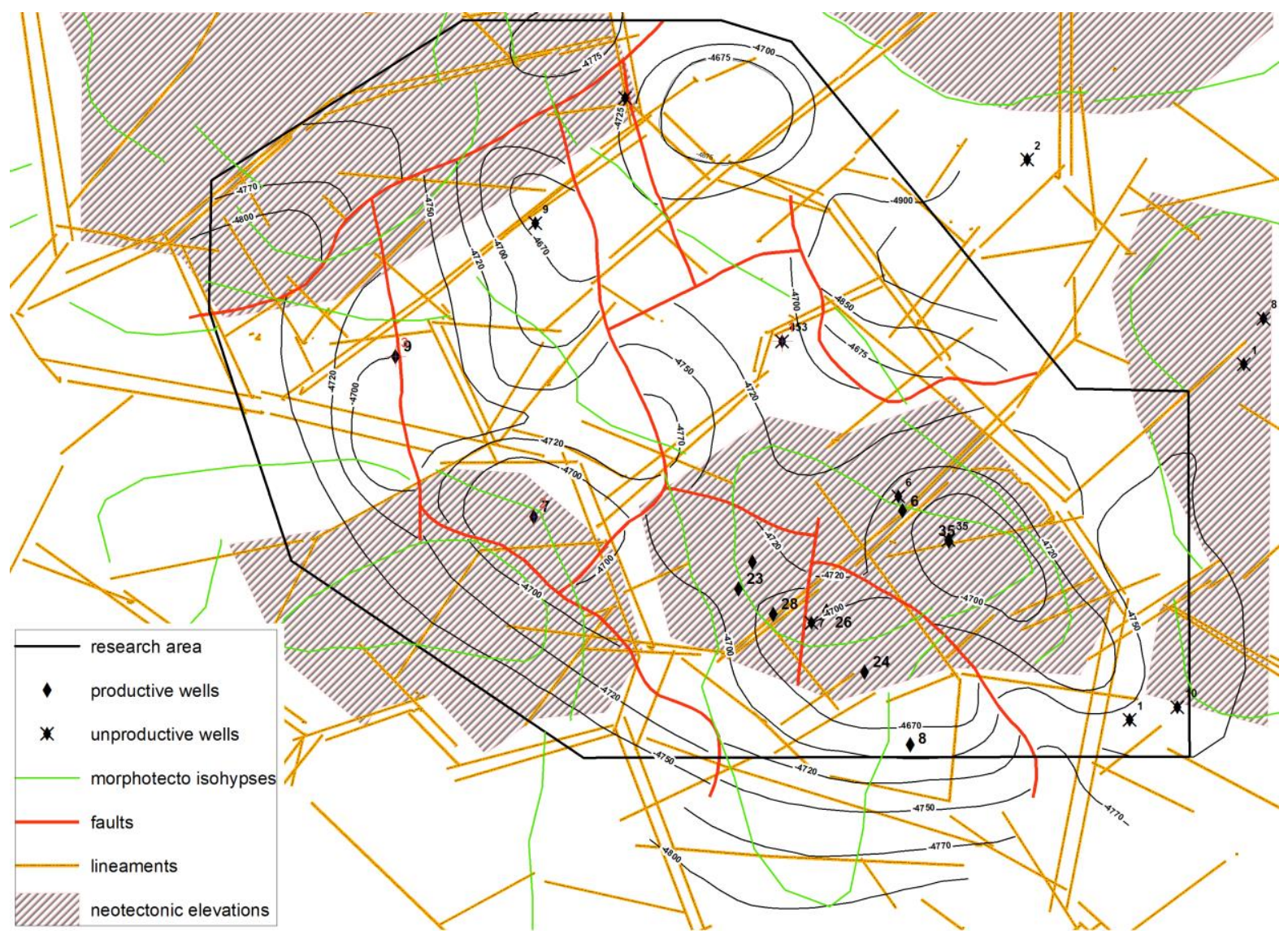

Fig. 4. Lypovodolynsk oil and gas field 
The coves of the structure is accentuated by the discordant laying of the right-hand tributary-moat of the river Horol, the top of which branches out, covering the raised relief block within the coves of the structure on both sides, forming a forked plan. This block is clearly fixed by the short left and right tributaries of the main moats flowing from the top of the block, which is emphasized by the plane flushing of the surface soils from the plane of the block and indicates its neotectonic activity. Similar in activity block is observed northwest of well No.9, from which it is separated by a lineament.

The initial data for integration at the Lypovodolynsk deposit are the spatial layers contained in the corresponding files of various formats and coordinate systems:

- Landsat/ETM+ satellite images, 6 spectral bands;

- SRTM terrain v3 digital terrain elevation data;

- land surface temperature;

- lineament density raster maps, 4 pcs. in $45^{\circ}$ increments;

- vector map of structural horizons;

- Neotectonic block map;

- map of geochemical anomalies;

- areas of optical anomalies of vegetation;

- location of productive / non-productive wells;

- map of depth of the terrain breakdown.

The estimation of oil and gas potential by the developed method was carried out on the basis of data from 10 information layers $(L=10)$. Tuples of primary data (see expression (12)) were formed by the expert method. Highly qualified geological experts were recruited; each of them had access to all information layers, was working independently and formed their own estimation hypotheses with assigning appropriate probabilities.

The problem of estimating oil and gas potential of the area under study was solved by two methods: by a method based on the Dempster-Shafer theory of evidence and described in [12] and by the proposed method.

The result of application of the method [12] for the forecasting oil and gas potential for different plots within the Lypovodolyn field is shown in Fig. 5. From its analysis, it is clear that for a large area of the deposit the value of the belief function is 0 .

This is due, first of all, to the fact that, as shown in [21], applying Dempster's combination rule in conflicting situations produces an unreasonable results, especially when belief masses of some hypotheses are close on zero.

The result of application of the proposed method is shown in Fig. 6. From its analysis it is clear that the entire area of the field is classified for the presence of hydrocarbons. Thus the proposed method is relieved of the disadvantages that are typical for Dempster's combination rule and allows to obtain not a blurred interval but an unambiguous point estimate.

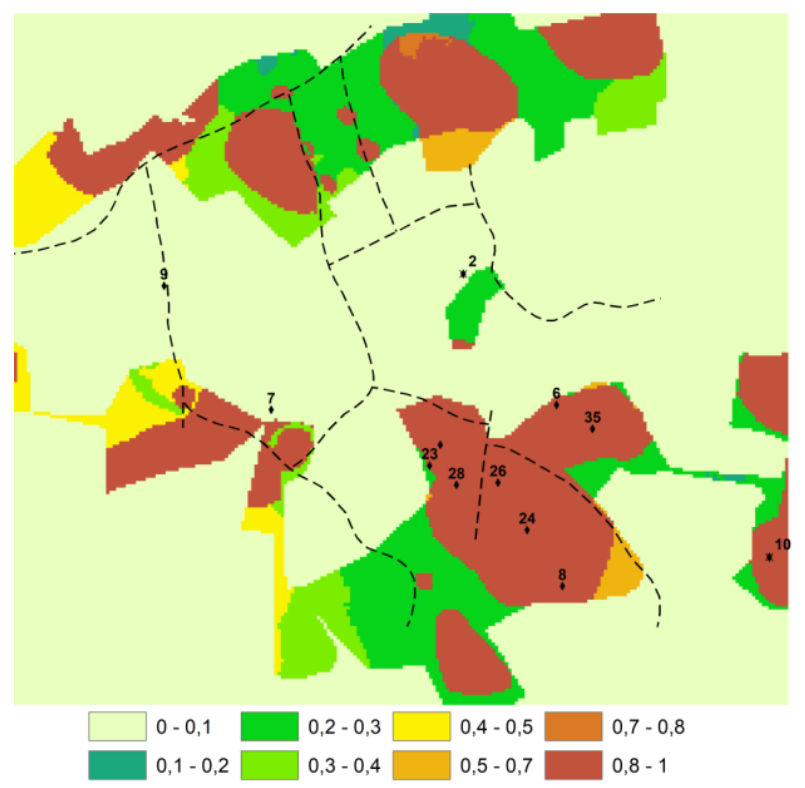

Fig. 5. The result of using Dempster-Shafer method [12] for the Lypovodolyn field

According to the results of the analysis of oil and gas content with the help of the proposed method the map of the distribution of output probabilities, by means of which it is possible to provide a complex evaluation of oil and gas potential of the studied area, has been plotted up (Fig. 6).

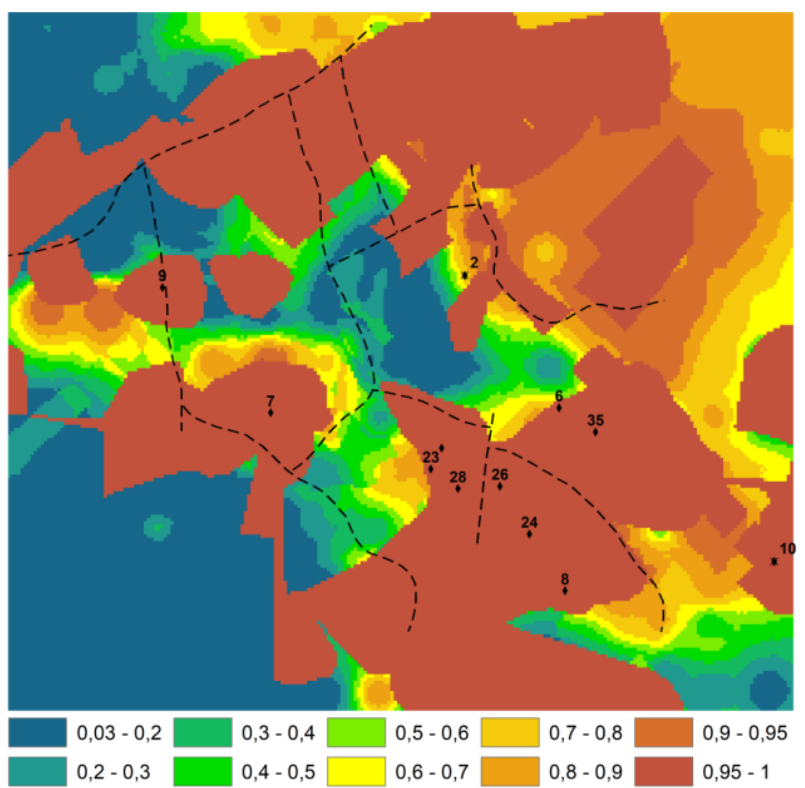


Fig. 6. The result of using the proposed method for the Lypovodolyn field

It should be noted that using the proposed method emphasizes the block heterogeneity of the deposit better, which is confirmed by geological exploration. Unproductive well No. 10 is classified as high predictive probability because it is at the edge of the neotectonic uplift. As for productive wells, they all relate to $80-90 \%$ forecast probability, as evidenced by drilling data.

\section{Conclusions}

The new approach and method to forecasting the oil and gas potential of subsoil plots is proposed. This approach actively exploits the mathematical apparatus of subjective logic.

The advantages of the proposed method are that it allows to adjusting the subjectivity and uncertainty of expert judgments, to generate adequate decisions on the results of the analysis one-time or rare events, and to avoid the majority of logical-computational problems that usually arise when combining conflicting hypothesis and opinions.

The proposed method was tested under the Lypovodolyn oil and gas condensate field. According to the results, the proposed method showed advantages over the method based on the Dempster-Shafer theory of evidence.

It is assumed that further research and efforts of authors will be focused on the technologization of the proposed method, the improvement of its algorithmic support and the writing of recommendations for its using in oil and gas exploration works.

\section{References}

[1] Demand for Oil \& Gas Grows Faster than Production in Most of the World, New Report Shows, International Association of Oil \& Gas Producers, URL: https://www.iogp.org/blog/ press-releases/global-production-report-pressreleases/demand-for-oil-gas-grows-fasterthan-production/

[2] S. Gaci, O. Hachay (Eds), Oil and Gas Exploration: Methods and Application, Wiley, 2017, 304 p., DOI: 10.1002/9781119227519

[3] V.V. Shestopalov, A.E. Lukin, V.A. Zgonnik, A.N. Makarenko, N.V. Larin, A.S. Boguslavsky, Essays on Earth Radioenvironmental (in Russian), NAS of Ukraine, 2019, 632 p.
[4] G. Wang, Q. Weng (Eds), Remote sensing of natural resources, CRC Press, 2014, 532 p., DOI: $10.1201 / \mathrm{b} 15159$

[5] S. Rashwan, M.A. Ismail, S. Fouad, Possibilistic Fusion for Landcover Mapping using Correlated Satellite Imagery for Environmental Change / Proceedings of the $5^{\text {th }}$ WSEAS Int. Conf. on Computational Intelligence, Man-Machine Systems and Cybernetics, Venice, Italy, November 20-22, 2006. P. 29-33.

[6] G. Sirbiladze, B. Ghvaberidze, P. Dvalishvili, Modeling Decision in the Dempster-Shafer Belief Structure Uncertainty / Proceedings of the $11^{\text {th }}$ WSEAS International Conference on Automatic Control, Modelling and Simulation, 2009. P. 297-302.

[7] A. Jøsang, Subjective Logic: A Formalism for Reasoning under Uncertainty, Springer, 2016, 337 p. DOI: 10.1007/978-3-319-423371

[8] J. Liebowitz, The Handbook of Applied Expert Systems, CRC Press, 1997. 736 p. DOI: 10.1201/9780138736654

[9] P. Wally, Statistical Reasoning with Imprecise Probabilities, Chapman and Hall, $1991.720 \mathrm{p}$.

[10] V.N. Evdokimenkov, D.M. Trofimov, M.K. Shuvaeva, Modern Methods and Algorithms for Processing Space, Geological, Geophysical and Geochemical Information to Forecast the Hydrocarbon Potential of Unexplored Subsoil Sections (in Russian), PhysMathLit, 2012. 320 p.

[11] K. Sukhanov, Approximation of Real Data by Fuzzy Sets for the Classification Problem (in Russian), Ukrainian Journal of Remote Sensing, 2019, No. 22, P. 22-26, DOI: 10.36023/ujrs.2019.22.154

[12] V.I. Lyalko, A.J. Hodorovsky, A.A. Apostolov, A.B. Vostokov, Quantitative Prognosis of Oil and Natural Gas Fields, ISPRS Archives, 2010, Vol. XXXVIII, Part 7B, P. 622-627, URL: https://www.isprs.org/proceedings/XXXVIII/ part7/b/pdf/622_XXXVIII-part7B.pdf

[13] M.O. Popov, M.V. Topolnitsky, O.V. Titarenko, Remotely-Sensed and Geologic and Geophysical Data Integration Using Enhanced Dempster-Shafer Method, In: V. Lyalko, M. Popov (Eds.), Novel Remote Sensing Methods for Minerals Prospecting, Scientific Centre for Aerospace Research of the Earth, 2017. P. 100-103,

URL: https://ujrs.org.ua/ujrs/article/view/ 129/145 
[14] G. Shafer, A Mathematical Theory of Evidence, Princeton University Press, 1976, $314 \mathrm{p}$.

[15] A. Jøsang, A Logic for Uncertain Probabilities, International Journal of Uncertainty, Fuzziness and Knowledge-Based Systems, 2001, Vol. 9, No. 3, P. 279-311, DOI: 10.1142/S0218488501 000831

[16] E.H.R. Fadda, M. Kakish, T.A. al Azab, The implementation of satellite images and associated digital image processing in addition to GIS modelling for urban mapping in Amman area, Jordan / WSEAS Transactions on Communications. Issue 2, Vol. 8, February 2009. P. 300-309.

[17] J. Dezert, D. Han, Z. Liu, J.-M. Tacnet, Hierarchical DSmP Transformation for Decision-Making under Uncertainty, Advances and Applications of DSmT for Information Fusion, 2015, Vol. 4. P. 125-132, DOI: 10.5281/ zenodo. 22469

[18] A. Jøsang, The Consensus Operator for Combining Beliefs, Artificial Intelligence, 2002, Vol. 141, No. 1-2, P. 157-170, DOI: 10.1016/S0004-3702(02)00259-X

[19] M.M. Ivanyuta, Y.O. Fedyshyn, B.I. Deneha, Y.O. Arsiriy, Y.G. Lazaruk, Atlas of Oil and Gas Fields of Ukraine (in Ukrainian), Vol. 3, Ukrainian Oil and Gas Academy, 1998, P. 273-282.

[20] M. Dudnikov, Prospects for Oil and Gas in the Southeastern Part of the Dnieper-Donets Basin, Visnyk Taras Shevchenko National University of Kyiv: Geology, 2012, No 58. P. 36-40,

URL: http://www.library.univ.kiev.ua/ukr/host/10.23 $.10 .100 / \mathrm{db} / \mathrm{ftp} / \mathrm{visnyk} / \mathrm{geolog} 58$ 2012.pdf

[21] L.A. Zadeh, On the Validity of Dempster's Rule of Combination, Memo M79/24, University of California, 1979, 15 p., URL: https://www2.eecs.berkeley.edu/Pubs/ TechRpts/1979/ERL-m-79-24.pdf

[22] L.G. Polpitiya, K. Premaratne, M.N. Murthi, D. Sarkar, Linear Time and Space Algorithm for Computing All the Fagin-Halpern Conditional Beliefs Generated from Consonant Belief Functions / Proceedings of The Thirty-Second International Florida Artificial Intelligence Research Society Conference (FLAIRS-32), Miami, Florida, USA, 2019. P. 482-487. ww.aaai.org

\section{Creative Commons Attribution License 4.0 (Attribution 4.0 International , CC BY 4.0)}

This article is published under the terms of the Creative Commons Attribution License 4.0 https://creativecommons.org/licenses/by/4.0/deed.e $\underline{\mathrm{n} U S}$ 